\title{
A Mutation in FBN1 Disrupts Profibrillin Processing and Results in Isolated Skeletal Features of the Marfan Syndrome
}

\author{
Dianna M. Milewicz, * Jami Grossfield, * Shi-Nian Cao, * Cay Kielty, ${ }^{\ddagger}$ Wesley Covitz, ${ }^{\mathbf{5}}$ and Tamison Jewett ${ }^{\mathbf{5}}$ \\ ${ }^{*}$ Department of Internal Medicine, University of Texas-Houston Medical School, Houston, Texas 77030; ${ }^{\ddagger}$ School of Biological Sciences, \\ University of Manchester, UK, M 13 9PT; and ${ }^{8}$ Department of Pediatrics, Bowman-Gray School of Medicine, Winston-Salem, \\ North Carolina, 27157
}

\begin{abstract}
Dermal fibroblasts from a 13-yr-old boy with isolated skeletal features of the Marfan syndrome were used to study fibrillin synthesis and processing. Only one half of the secreted profibrillin was proteolytically processed to fibrillin outside the cell and deposited into the extracellular matrix. Electron microscopic examination of rotary shadowed microfibrils made by the proband's fibroblasts were indistinguishable from control cells. Sequencing of the $F B N 1$ gene revealed a heterozygous $C$ to $T$ transition at nucleotide 8176 resulting in the substitution of a tryptophan for an arginine $(\mathbf{R 2 7 2 6 W})$, at a site immediately adjacent to a consensus sequence recognized by a cellular protease. Six other individuals in the proband's family had the $F B N 1$ mutation that segregated with tall stature. None of the affected individuals have cardiac or ocular manifestations of the Marfan syndrome. This mutation identifies a putative site for profibrillin to fibrillin processing, and is associated with isolated skeletal features of the Marfan syndrome, indicating that the $F B N 1$ gene is one of the genes that determines height in the general population. The cellular effect of the mutation may be equivalent to a "null" $F B N 1$ allele and may define the phenotype associated with $F B N 1$ "null"' alleles. (J. Clin. Invest. 1995. 95:2373-2378.) Key words: fibrillin • extracellular matrix • elastin-associated microfibrils • proteolytic processing • null allele
\end{abstract}

\section{Introduction}

The Marfan syndrome is an autosomal dominant disorder with cardiovascular, ocular, and skeletal manifestations $(1,2)$. The cardiovascular complications of the disorder include aortic root dilatation and dissection, mitral valve regurgitation, and mitral valve prolapse. These complications can lead to premature death

Address correspondence to Dianna M. Milewicz, M.D., Ph.D., Department of Internal Medicine, University of Texas-Houston Medical School, MSB 1.614, 6431 Fannin, Houston, TX 77030. Phone: 713792-0727; FAX: 713-792-5187.

Received for publication 9 November 1994 and in revised form 6 February 1995.

J. Clin. Invest.

(c) The American Society for Clinical Investigation, Inc.

0021-9738/95/05/2373/06 \$2.00

Volume 95, May 1995, 2373-2378 if left untreated (3). The major ocular manifestations are ectopia lentis and myopia. The skeletal features are readily apparent when examining affected individuals and include tall stature secondary to dolichostenomelia, arachnodactyly, scoliosis, and pectus deformities. Although any of the manifestations of the Marfan syndrome can occur as an isolated finding in an individual, it is the constellation of findings involving these systems and the autosomal dominant inheritance of these findings that characterize the Marfan syndrome (4).

Extensive research has established that mutations in the FBN1 gene result in the Marfan syndrome (5-8). FBN1 encodes a large glycoprotein, fibrillin, that is a component of microfibrils found in the extracellular matrix (9). The majority of mutations identified in individuals with the Marfan syndrome are missense mutations affecting primarily the numerous domains with homology to epidermal growth factor (EGF) found throughout the protein $(5,7,8,10-12)$. Nonsense mutations, splicing errors and small genomic deletions predicted to result in truncated fibrillin- 1 molecules have been identified $(8,13$, 14). No mutations predicted to lead to a nonexpressed FBN1 allele have been identified as a cause of the Marfan syndrome.

Biochemical studies have delineated the normal synthesis and cellular processing of fibrillin by fibroblasts (15). Fibrillin is made as a proprotein (profibrillin) that is $\sim 350 \mathrm{kD}$. Profibrillin is secreted and then proteolytically processed to a lower molecular weight form, $\sim 20 \mathrm{kD}$ smaller. The processed fibrillin is then deposited into the pericellular matrix in the form of microfibrils. Fibroblasts explanted from the majority of Marfan patients $(>85 \%)$ will show defects in the amount of profibrillin synthesized, the efficiency of secretion of profibrillin, or decreased incorporation of fibrillin into the pericellular matrix. When the structure of fibrillin-containing microfibrils from Marfan cell strains are studied using rotary shadowing electron microscopy, abnormal microfibril structure is evident (16).

$F B N 1$ gene mutations have been found to be responsible for a spectrum of conditions phenotypically related to the Marfan syndrome, including dominantly inherited ectopia lentis, severe neonatal Marfan syndrome, and the MASS phenotype, which refers to individuals or families with mitral valve prolapse, nonprogressive aortic root dilatation, and skin and skeletal features of the Marfan syndrome $(7,8,11,17-19)$. We describe a three generation family with an FBN1 gene mutation that results in isolated skeletal features of the Marfan syndrome. No one in the family has cardiovascular or ocular complications characteristic of the Marfan syndrome. In the majority of affected individuals in the family the only skeletal manifestation is tall stature ( $>$ 95th percentile for age and sex). The FBN1 mutation disrupts the extracellular processing of profibrillin to fibrillin, iden- 


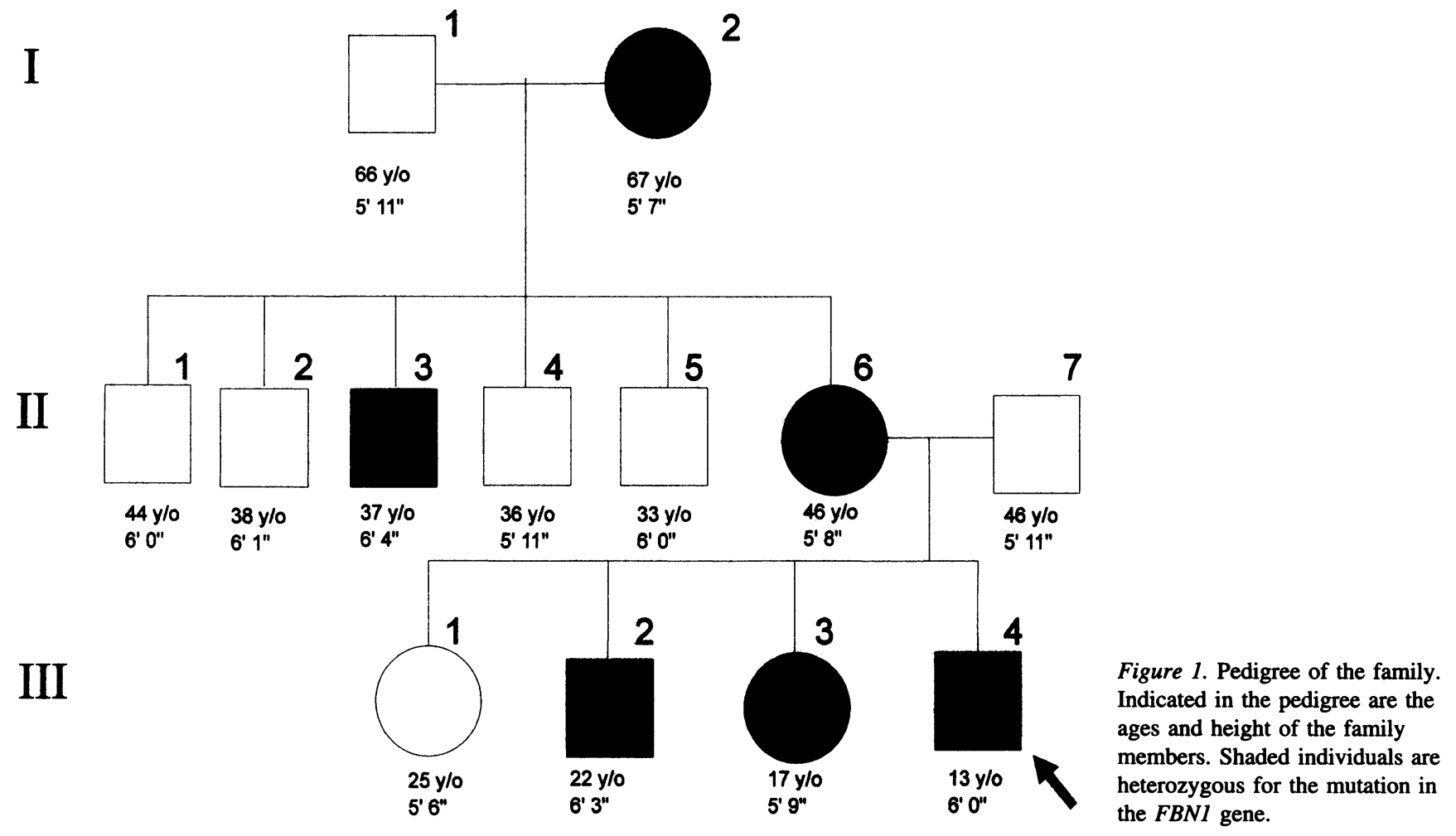

tifying the putative cleavage site of this processing. The unprocessed profibrillin is not incorporated into microfibrils. Therefore, this mutation may be equivalent at the cellular level to a null or nonexpressed $F B N 1$ allele and may define a phenotype associated with lack of expression of one $F B N I$ allele.

\section{Methods}

Clinical summary. The proband was a 13-yr-boy with tall stature (height $6 \mathrm{ft}$. [ $>95$ th percentile for age], arm span equal to height, upper to lower segment ratio 0.89 ), pectus carinatum, scoliosis, arachnodactyly, and pes planus. The proband had a normal echocardiogram (proximal ascending aorta $16 \mathrm{~mm}$ ) and ophthalmologic exam revealed accommodative esotropia without myopia or ectopia lentis. The pedigree of the proband's family is shown in Fig. 1. The height and age of the family members is indicated. No other family members were known to have pectus deformities or scoliosis. The proband's brother (III-2) had a normal echocardiogram (proximal ascending aorta $23 \mathrm{~mm}$ ) and ophthalmologic examination. He had an upper to lower segment ratio of 1.0, his arm span equaled his height, and his metacarpal index was 8.5 , two standard deviations above the mean. The proband's mother (II-6) and maternal grandmother (I-2) had normal echocardiographic examinations (proximal ascending aorta 29 and $30 \mathrm{~mm}$, respectively). The maternal grandmother had 8 siblings, 6 of which are alive and between 60 and 80 years of age. One brother died at the age of 18 of polio and a sister died at the age of 76 of cancer.

Preparation and electrophoretic analysis of fibrillin. Dermal fibroblasts were obtained from the proband after appropriate consent. Fibroblasts were explanted and maintained in culture as previously described (15). To radiolabel synthesized proteins, 250,000 dermal fibroblasts were plated in 35-mm dishes, allowed to attach and spread for $72 \mathrm{~h}$ in Dulbecco-Vogt modified Eagle' medium (DME) supplemented with $10 \%$ fetal calf serum. Pulse-chase studies were done using $\left[{ }^{35} \mathrm{~S}\right]-$ cysteine to radiolabel synthesized proteins and the media, cell lysate, and cell layer were harvested separately using a previously described protocol (15). Proteins were dissolved in sample buffer containing so- dium dodecyl sulfate (SDS) and $\beta$-mercaptoethanol, separated by electrophoresis on a $4 \%$ acrylamide slab gel containing SDS, and visualized by autoradiography. The location of fibrillin in this gel system has been previously established (15).

Identification of the mutation. Total genomic DNA was harvested from blood by using a previously described protocol (20). Exon 64 of the $F B N 1$ gene was amplified from genomic DNA using primers complimentary to sequences in intron 63 (I63S, $5^{\prime}$-CCTACCTTGTCTTCCCATTCTAATG-3') and 64 (I64AS, 5'-ACAGGAGACATCAGGAGAAAC-3'), and denaturing at $94^{\circ} \mathrm{C}$ for $30 \mathrm{~s}$, annealing at $58^{\circ} \mathrm{C}$ for $30 \mathrm{~s}$ and extending at $72^{\circ} \mathrm{C}$ for $30 \mathrm{~s}$. The DNA fragment was purified and sequenced on an ABI Applied Biosystems automated sequencer model 373A (Applied Biosystems, Inc., Foster City, CA).

Population screening for the mutation. Total genomic DNA was harvested from 50 unrelated individuals. Additionally, genomic DNA was harvested from dermal fibroblast cell strains from 19 controls and 23 Marfan patients. The amplified DNA fragments were digested with BscGI generously supplied by Dr. Carole Keating at New England Biolabs. The resulting DNA fragments were separated by electrophoresis through $6 \%$ polyacrylamide gel and visualized using ethidium bromide.

Ultrastructural analysis of microfibrils. Protocols used for ultrastructural analysis of the microfibrils have been previously described (16). Briefly, for microfibril extractions, cells were maintained at postconfluence for up to $3 \mathrm{wk}$. Cell layers were washed in $50 \mathrm{mM}$ Tris$\mathrm{HCl}, \mathrm{pH} 7.4$, containing $0.4 \mathrm{M} \mathrm{NaCl}, 5 \mathrm{mM} \mathrm{CaCl}_{2}, 0.1 \mathrm{mg} / \mathrm{ml}$ bacterial collagenase (type IA), $2 \mathrm{mM}$ PMSF, and $5 \mathrm{mM}$ NEM. Soluble extracts were clarified by centrifugation for $15 \mathrm{~min}$ at $7,500 \mathrm{~g}$ on a bench microfuge before size fractionation by gel filtration chromatography on a Sepharose CL-2B column at room temperature. Void volume fractions were visualized for their microfibril content by rotary shadowing using a modification of the mica sandwich technique (21).

\section{Results}

Abnormal processing of profibrillin to fibrillin by the proband's dermal fibroblasts. We have previously shown that fibrillin is 


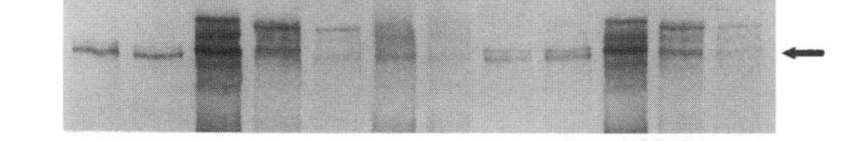

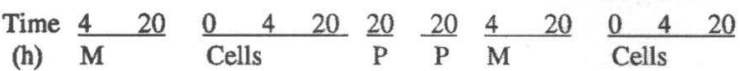

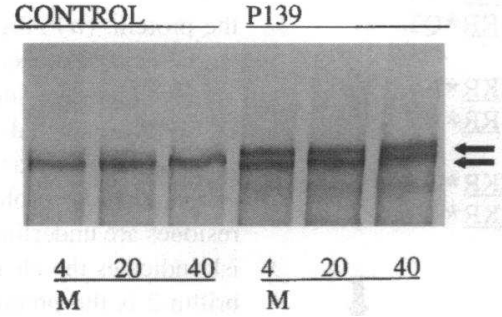

Figure 2. Abnormal processing of profibrillin to fibrillin by the proband's dermal fibroblasts. Cells from the proband (P139) and an agematched control were incubated with $\left[{ }^{35} \mathrm{~S}\right]$ cysteine for $30 \mathrm{~min}$ and then chased for up to $40 \mathrm{~h}$ without label present. $M$, media, Cells, cell lysate, and $P$, extracellular matrix were harvested separately at varying periods of time. $(A)$ Fibrillin is synthesized within the cells as profibrillin (arrow, Cells, $0 \mathrm{~h}$ ). It is then secreted from the cell within the $20 \mathrm{~h}$ of the chase $($ Cells, $20 \mathrm{~h})$. Once outside the cells, profibrillin is converted to fibrillin by the control cells $(M, 4$ and $20 \mathrm{~h})$. The proband's cells maintain an equal pool of profibrillin and fibrillin in the media out to $20 \mathrm{hrs}(M, 20 \mathrm{~h})$. Only the processed form of fibrillin is deposited in the extracellular matrix by both cell strains $(P, 20 \mathrm{~h})$. $(B)$ The media only was harvested at varying periods of time up to $40 \mathrm{~h}$ to demonstrate that the equal pool of profibrillin and fibrillin was maintained by the proband's cells (upper and lower arrows, respectively), whereas the control's cells completely converted profibrillin to fibrillin (lower arrow).

synthesized by dermal fibroblasts in a precursor form (profibrillin) with an approximate molecular weight of $350 \mathrm{kD}$, then secreted from the cell and processed to a lower molecular weight product (fibrillin) (Fig. 2 A) (15). The rate of conversion of the precursor to final product in the media varies among cell strains but is generally complete by $20 \mathrm{~h}$. This proteolytically processed fibrillin is then deposited in the extracellular matrix surrounding the cells, presumably in the form of microfibrils.

The amount of profibrillin synthesized by the proband's cells and the efficiency of secretion of profibrillin was normal when compared to age-matched control cells (Fig. $2 A$ ). Once outside the cells, profibrillin was essentially completely converted to fibrillin by $20 \mathrm{~h}$ by the control cells. In contrast, only one-half of the profibrillin was processed to fibrillin by proband's cells by $20 \mathrm{~h}$ and this pool of profibrillin remained unprocessed for up to $40 \mathrm{~h}$ (Fig. $2 \mathrm{~B}$ ). Only the processed form of fibrillin was deposited in the extracellular matrix by both the proband's and control cells (Fig. $2 A$ ). The amount of fibrillin incorporated into the extracellular matrix by the proband's cells was approximately one-half that of the control cells.

Identification of a FBNI mutation at the proteolytic processing site for profibrillin to fibrillin. Exon 64 of the FBNI gene encodes a region of the unique carboxy terminal domain of fibrillin that contains a known recognition motif for proteolytic cleavage by a cellular protease. Therefore, exon 64 of the FBN1 gene was amplified from the proband's genomic DNA and sequenced directly to identify a possible mutation affecting the proteolytic processing of profibrillin. DNA from the proband was heterozygous for a $\mathrm{C}$ to $\mathrm{T}$ transition at nucleotide 8176 (22). This mutation changes an arginine to a tryptophan at nucleotide codon 2726 , a site directly adjacent to the consensus cleavage sequence for a cellular protease (Fig. $3 a$ ). This cleavage is found in the unique carboxyl-terminus of the fibrillin protein (Fig. $3 b$ ).

Exon 64 was amplified and sequenced directly using genomic DNA from all 13 family members shown in Fig. 1. Sequencing of exon 64 identified five additional individuals in the family who were heterozygous for the mutation, I-2, II-3, II-6, III-2, III-3. The $\mathrm{C}$ to $\mathrm{T}$ transition was not found in exon 64 of the FBN1 gene from 50 unrelated controls. Additionally, the mutation was not present in DNA from 42 cell strains screened for the transition. These cell strains were obtained from 23 Marfan patients and 19 unaffected individuals and had previously been shown to have normal processing of profibrillin to fibrillin (15).

Ultrastructural analysis of the microfibrils made by the proband's cells. Examination of high relative molecular mass material from the proband's cells by rotary shadowing electron microscopy showed that the cells elaborated long, abundant microfibrils that were similar in morphology to those isolated from control cells (Fig. 4) $(16,21)$. The morphology of microfibrils consists of beaded domains with a periodicity of $50-55 \mathrm{~nm}$ and interbeaded domains. Microfibrils made by the proband's cells had normal periodicity of the beaded domains. The interbeaded domains appeared indistinct, but within the normal variation of microfibrillar appearance by this preparative process.

\section{Discussion}

The family described here is the first family identified with an FBN1 mutation that segregates with isolated skeletal features of the Marfan syndrome. The proband has dolichostenomelia, arachnodactyly, scoliosis, and pes planus. The remainder of the affected family member are tall ( $>$ 95th percentile for age and sex ). Four affected family members have had normal echocardiograms, including the 67-yr-old maternal grandmother. Additionally, two affected individuals have had normal ophthalmologic examinations. No one in the family has been diagnosed with the Marfan syndrome or is known to have any of the specific diagnostic findings associated with the Marfan syndrome, i.e., aortic aneurysms or dissection, mitral valve abnormalities, lens dislocation, or dural ectasia (4). These observations indicate that $F B N 1$ mutations can cause isolated skeletal features, implicating the $F B N 1$ gene as one of the genes that determines height in the general population.

The mutation in the FBNI gene in this family has an interesting effect on fibrillin processing. Fibrillin is synthesized as a proprotein in the cell (profibrillin), secreted and proteolytically processed outside the cell to fibrillin by removal of $\sim 20 \mathrm{kD}$ of the protein. This mutation disrupts the normal processing of one-half of the secreted profibrillin to fibrillin, implying the mutation disrupts the proteolytic processing site in the profibrillin molecule. The amino acid altered by the mutation (R2726W) occurs immediately adjacent to a known tetrabasic consensus 
a

Fibrillin

Mutated Fibrillin (P139)

Fibrillin-2

Plasma Proteins

Human von Willebrand Factor Precursor

Human Factor VII Precursor

Human Complement C3 Precursor

Receptors

Human Insulin Proreceptor

Human IGF-1 Proreceptor

Human Platelet Glycoprotein IIb Precursor

Viral Envelope Glycoproteins

HIV-1 gp160

HTLV-1 gp4 6/p20

Virulent Influenza Virus Hemagglutinins

FPV/34 (H7)

Tern/SA/61(H5)
PEPTIDE SEQUENCE FLANKING

TETRABASIC CLEAVAGE 8 ITE

$-6-4-1 * 1$

PKRGRKRR*ST

PKWGRKRR*ST

KKDSRQKR *SI

PLSHR्RKRR*SL

GVLHRRRR *AN

QPAARRRR *SV

PRPSRKKK * SL

PRPERKRR *DV

$A H H K \underline{R D R R} * Q I$

RVVQREKR $* A V$

TLGSRSRR $\underline{R}$ AV

PSKKRE $\underline{K R} * G$ RETRREKR $* G$

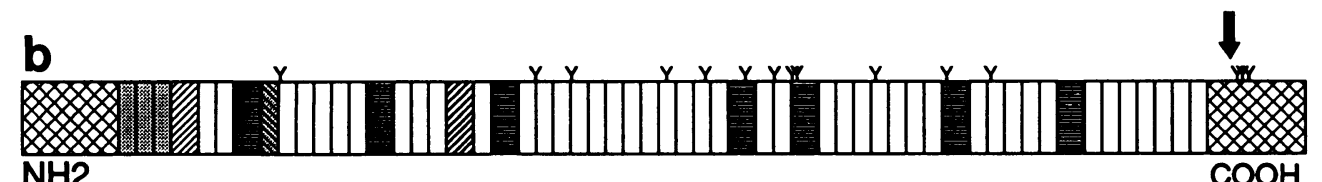

$\mathrm{NH} 2$
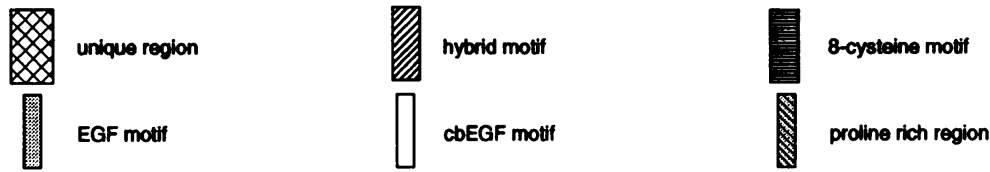

Figure 3. Sequence of the putative proteolytic processing site of profibrillin and location of the site in the protein. (a) Alignment of the amino acid sequences around the putative cleavage site of profibrillin and the mutated profibrillin from the proband as well as and other precursor proteins. Basic residues are underlined. The asterisk indicates the cleavage site. Fibrillin-2 is the product of the $F B N 2$ gene. $I G F-I$ insulin-like growth factor-1; HIV, human immunodeficiency virus, $H T L V-1$, human $\mathrm{T}$ cell leukemia virus type I. (B) Graphic representation of the structural motifs found in the fibrillin protein, the location of the putative processing site (arrow) and potential sites for $\mathrm{N}$-linked glycosylation $(Y)$. site for cellular proteases that would be predicted to remove a 19-kD fragment from the protein (Fig. 3).

Many secreted and membrane proteins are produced from precursors through cleavage at multiple basic residues, which occurs during secretion of the protein from the cell. Mono-, di-, tri-, tetra-, and pentabasic cleavage motifs are found in higher eukaryotes and their viral pathogens. The most common processing site found in mammals is the dibasic motif, Lys.Arg or
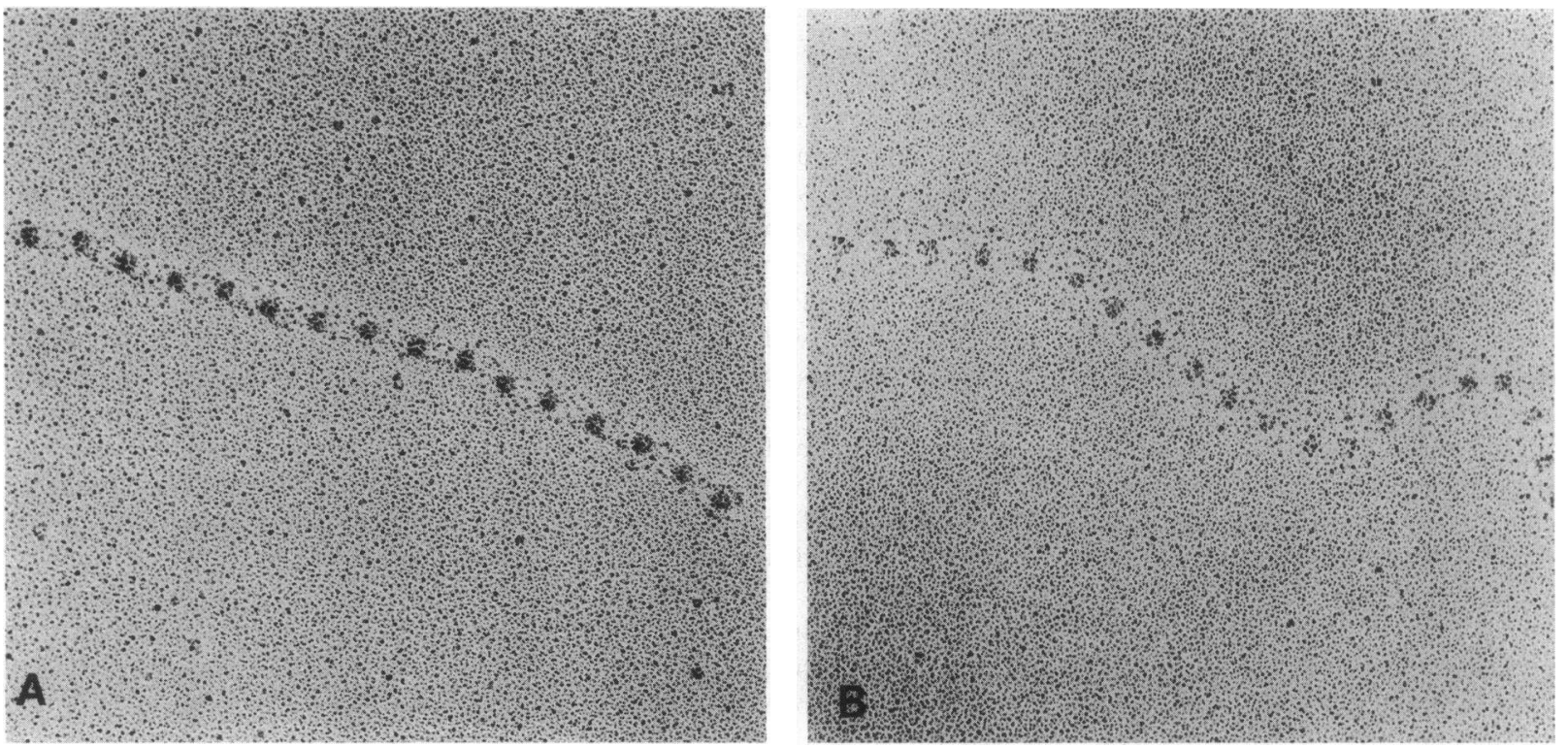

Figure 4. Microfibrils formed by control cells $(A)$ and the proband's cells $(B)$. The morphology of microfibrils consists of beaded domains and interbeaded domains. The periodicity of the beaded domains was the same between the microfibrils from the control and proband's cells. Although the interbeaded domains of microfibrils from the proband's cells appeared indistinct, this is within normal variation by this preparative process. $\times 120,000$. 
Arg.Arg, together with the motif that adds an additional basic amino acid, Lys/Arg.X.Lys/Arg.Arg (Fig. $3 a$ ). Subsets of this type of motif, the tri- or tetrabasic site, have an additional 1 or 2 basic residues. Proteolytic cleavage occurs immediately carboxy terminal to the tetrabasic signal. Mutagenesis studies and naturally occurring variants of the tetrabasic recognition site have indicated the critical importance of the basic amino acids at the -1 and -4 positions from the cleavage point; replacement of these residues with non-basic amino acids can fully inhibit processing (23-25). The presence of a basic residue in the -2 position is not always critical $(23,25)$. Further information about the structural requirements for cleavage have been obtained from extensive studies of the proteolytic processing of influenza virus hemagglutinin (HA), a viral protein that contains a tetrabasic cleavage motif. These studies suggest that these proteases require not only the presence of the basic amino acids at the cleavage site but also a structural feature distinct from the basic residues at the connecting peptide (2628 ). Removal or replacement of basic amino acids at positions -5 and -6 result in diminished cleavage of HA $(26,28)$. Although the FBN1 mutation described here does not disrupt the four basic amino acids at the cleavage site, it replaces a basic amino acid at the -6 position, arginine, with tryptophan, a residue with a bulky side group. Thus, if the tetrabasic consensus sequence in the carboxy terminal domain is indeed the site of proteolytic processing of profibrillin, this mutation would be predicted to alter the cleavage site such that the protease would not cleave the profibrillin. Of interest is the fact that fibrillin2 , the protein product of a closely related gene, $F B N 2$, has a tribasic sequence at this same location (Fig. $3 a$ ) (29).

The cellular protease responsible for processing profibrillin to fibrillin has not been identified. A number of cellular proteases that recognize the basic amino acid cleavage motif have been characterized. Furin (or PACE for Paired basic Amino acid Cleaving Enzyme) was the first characterized protease in mammalian cells and it has homology to the yeast KEX2 serine protease and the bacterial subtilisin $(30,31)$. Furin is a membrane associated, calcium dependent serine protease. It has been localized to the trans-Golgi but recent evidence indicates it cycles between the cell surface and the Golgi apparatus (32) and that it may act on extracellular proteins through its proteolytic activity on the cell surface (33). The pulse-chase protocol used to metabolically label profibrillin indicates that profibrillin is not proteolytically processed until after it is secreted from the cell (15). The processed fibrillin appears immediately after secretion of profibrillin from the cell and is complete within 20 $h$. If furin is the protease processing profibrillin to fibrillin, it is surprising that the profibrillin would not be processed in the Golgi compartment during secretion from the cell. These observations indicate if furin is responsible for profibrillin processing, then profibrillin must contain a signal that prevents intracellular processing during secretion and allows only extracellular processing. Alternatively, furin may not be the protease responsible for profibrillin processing and the protease responsible acts on profibrillin only in the extracellular space.

Examination of the extracellular matrix formed by the proband's cells indicate that only the proteolytically processed fibrillin is incorporated into the matrix, despite the fact that the profibrillin remains as a stable pool in the media for up to 40 h (Fig. 2 A). Additionally, electron microscopic studies of the structure of the microfibrils formed by the proband's cells indicate that these microfibrils do not differ from those formed by control cells. These analyses indicate that the profibrillin is not incorporated into microfibrils and does not appear to disrupt the structural integrity of the microfibrils formed. These facts raise the possibility that this mutation may allow the deposition of one-half the normal amount of fibrillin into structurally normal microfibrils. At the protein level this mutation may be equivalent to a nonexpressed or "null" FBN1 allele. There are over 30 published $F B N 1$ mutations identified as the cause of Marfan syndrome in individuals, the majority of which are point mutations affecting the epidermal growth factor-like domains (7, $8,11)$. Although many of these mutations affect the level of expression from the mutant allele, none of the mutations characterized completely suppress expression from one allele. Cell strains from Marfan patients make structurally abnormal microfibrils (16). This observation suggests the fibrillin produced from the abnormal allele affects microfibrillar structure despite the production of fibrillin from the normal allele, i.e., a dominant negative effect (34). Because the effect of the mutation described here on the fibrillin protein may be equivalent to a nonexpressed allele it may define the phenotype associated with a "null" allele of the FBN1 gene. Alternatively, the unprocessed profibrillin may disrupt the microfibrils that are formed in some way that we cannot identify by our assays thereby affecting the microfibrils made from the normal allele.

In a number of dominant disorders, a mutation that produces nonexpression of one allele causes a milder form of the disease than missense mutations because of dominant negative effect of mutations on protein function (34). Mutations in the type I collagen genes (COL1A1 and COL1A2) result in osteogenesis imperfecta (OI), with OI type II being the most severe form of the disease and OI type I the least severe (35). OI Type II results from missense mutations in the COL1A1 or COL1A2 gene that alter glycines found in the triple helical region of the type I collagen molecule. These mutations cause the production of a structurally abnormal type I collagen. The majority of cases of OI Type I result from mutations that affect the production from one of the COL1A1 alleles and are equivalent to a "null" allele for the COL1A1 gene. This leads to a $50 \%$ reduction in the level of type I collagen, but the collagen is structurally normal. Thus, a qualitative abnormality in the protein structure leads to a more severe phenotype than a quantitative abnormality. The FBNI gene mutation described here is associated with a mild phenotype and may describe the phenotype associated with a "null" $F B N 1$ allele.

A previously described mutation, W2756X, predicts a profibrillin molecule that is truncated 25 amino acids on the carboxyl terminal side of the proposed profibrillin cleavage site (13). The mutation results in inefficient secretion of profibrillin and disrupts microfibril structure, implying the abnormal fibrillin is incorporated into the matrix without being processed (Raghunath M., C. M. Kielty, and B. Steinmann, manuscript submitted for publication). Thus, the effect of this mutation on profibrillin processing and microfibril formation is very different from the mutation described here.

In summary, these studies demonstrate that $F B N 1$ gene mutations can cause isolated skeletal features of the Marfan syndrome in the absence of ocular and cardiovascular complications. FBN1 gene mutations have also been shown to be responsible for other related syndromes, such as dominantly inherited ectopia lentis. Although these studies further our understanding of the disease states associated with $F B N 1$ gene mutations, the results complicate genetic counseling of individuals with $F B N 1$ 
gene mutations. Until we further understand how FBN1 mutations produce a particular phenotype, we cannot determine with certainty which individuals with $F B N 1$ gene mutations will have the life-threatening cardiovascular complications associated with the Marfan syndrome.

\section{Acknowledgments}

We would like to thank the family for their cooperation and interest in this study. We are indebted to Dr. Carole Keating at New England Biolabs for supplying the BscGI enzyme, Phyl Atcherson for helping to prepare the manuscript, and the University of Texas Microbiology Core facility for timely and accurate technical assistance.

This work was supported in part by a Pfizer Scholars Award, a March of Dimes Basil O'Connor Award and the Joel E. Smilow Fund from the National Marfan Foundation to D. Milewicz.

\section{References}

1. Pyeritz, R. E., and V. A. McKusick. 1979. The Marfan syndrome: diagnosis and management. N. Engl. J. Med. 300:772-777.

2. Pyeritz, R. E. 1993. The Marfan syndrome. In Connective tissue and its heritable disorders. P. M. Royce and B. Steinmann, editors. Wiley-Liss and Sons, New York. 437-468.

3. Murdoch, J. L., B. A. Walker, B. L. Halpern, J. W. Kuzma, and V. A. McKusick. 1972. Life expectancy and causes of death in the Marfan syndrome. N. Engl. J. Med. 286:804-808.

4. Beighton, P., A. dePaepe, D. Danks, G. Finidori, T. Gedde-Dahl, R. Goodman, J. G. Hall, D. W. Hollister, W. Horton, V. A. McKusick et al. 1988. International nosology of heritable disorders of connective tissue, Berlin. Am. J. Med. Genet. 29:581-594.

5. Dietz, H. C., G. R. Cutting, R. E. Pyeritz, C. L. Maslen, L. Y. Sakai, E. G Corson, A. Puffenberger, A. Hamosh, J. Nanthakumar, S. M. Curristin et al. 1991 Marfan syndrome caused by a recurrent de novo missense mutation in the fibrillin gene. Nature (Lond.). 352:337-339.

6. Lee, B., M. Godfrey, E. Vitale, H. Hori, M. G. Mattei, M. Sarfarazi, P. Tsipouras, F. Ramirez, and D. W. Hollister. 1991. Linkage of Marfan syndrome and a phenotypically related disorder to two different fibrillin genes. Nature (Lond.). 352:330-334.

7. Tsipouras, P., R. DelMastro, M. Sarfarazi, B. Lee, A. H. Child, M. Godfrey, R. B. Devereux, D. Hewett, B. Steinmann, E. Vitale et al. 1992. Genetic linkage of the Marfan syndrome, ectopia lentis, and congenital contractural arachnodactyly to the fibrillin genes on chromosomes 15 and 5. N. Engl. J. Med. 326:905-909.

8. Dietz, H. C., I. McIntosh, L. Y. Sakai, G. M. Corson, S. C. Chalberg, R. E Pyeritz, and C. A. Francomano. 1993. Four novel FBN1 mutations: Significance for mutant transcript level and EGF-like domain calcium binding in the pathogenesis of Marfan syndrome. Genomics. 17:468-475.

9. Sakai, L. Y., D. R. Keene, and E. Engvall. 1986. Fibrillin, a new 350-kD glycoprotein, is a component of extracellular microfibrils. J. Cell Biol. 103:24992509.

10. Dietz, H. C., J. M. Saraiva, R. E. Pyeritz, G. R. Cutting, and C. A Francomano. 1992. Clustering of fibrillin missense mutations in Marfan syndrome patients at cysteine residues in EGF-like domains. Human Mutation 1:366-374.

11. Kainulainen, K., L. Karttunen, L. Puhakka, L. Sakai, and L. Peltonen. 1994. Mutations in the fibrillin gene responsible for dominant ectopia lentis and neonatal Marfan syndrome. Nature Genetics 6:64-69.

12. Stahl-Hallengren, C., T. Ukkonen, K. Kainulainen, U. Kristofersson, T. Saxne, K. Tornqvist, and L. Peltonen. 1994. An extra cysteine in one of the noncalcium-binding epidermal growth factor-like motifs of the FBN1 polypeptide is connected to a novel variant of Marfan syndrome. J. Clin. Invest. 94:709-713.

13. Kainulainen, K., L. Y. Sakai, A. Child, F. M. Pope, L. Puhakka, L. Ryhanen, A. Palotie, I. Kaitila, and L. Peltonen. 1992. Two mutations in Marfan syndrome resulting in truncated fibrillin polypeptides. PNAS. 89:5917-5921.

14. Godfrey, M., N. Vandemark, M. Wang, M. Velinov, D. Wargowski, P.
Tsipouras, J. Han, J. Becker, W. Robertson, S. Droste, and V. H. Rao. 1993. Prenatal diagnosis and a donor splice site mutation in fibrillin in a family with Marfan syndrome. Am. J. Hum. Genet. 53:472-480.

15. Milewicz, D. M., R. E. Pyeritz, E. S. Crawford, and P. H. Byers. 1992. Marfan syndrome: defective synthesis, secretion and extracellular matrix formation of fibrillin by cultured dermal fibroblasts. J. Clin. Invest. 89:79-86.

16. Kielty, C. M., and C. A. Shuttleworth. 1994. Abnormal fibrillin assembly by dermal fibroblasts from two patients with the Marfan syndrome. J. Cell Biol. 124:997-1004.

17. Milewicz, D. M., and M. Duvic. 1994. Severe neonatal Marfan syndrome resulting from a de novo three base pair insertion into the fibrillin gene on chromosome 15. Am. J. Hum. Genet. 54:447-453.

18. Glesby, M. J. and R. E. Pyeritz. 1994. Association of mitral valve prolapse and systemic abnormalities of connective tissue. JAMA (J. Am. Med. Assoc.). 262:523-528.

19. Pereira, L., O. Levran, F. Ramirez, J. R. Lynch, B. Sykes, R. E. Pyeritz, and H. C. Dietz. 1994. A molecular approach to the stratification of cardiovascular risk in families with Marfan's syndrome. N. Engl. J. Med. 331:148-153.

20. Sambrook, J., E. F. Fritsch, and T. Maniatis. 1989. Molecular Cloning. A Laboratory Manual. Cold Spring Harbor Laboratory Press, Cold Spring Harbor, New York.

21. Kielty, C. M., L. Berry, S. P. Whittaker, M. E. Grant, and C. A. Shuttleworth. 1993. Microfibrillar assemblies of foetal bovine skin. Developmental expression and relative abundance of type VI collagen and fibrillin. Matrix. 13:103-112.

22. Pereira, L., M. D'Alessio, F. Ramirez, J. Lynch, B. Sykes, T. Pangilinan, and J. Bonadio. 1993. Genomic organization of the sequence coding for fibrillin, the defective gene product in Marfan syndrome. Hum. Mol. Genet. 2:961-968.

23. Rehemtulla, A., and R. J. Kaufman. 1992. Preferred sequence requirements for cleavage of Pro-von Willebrand factor by propeptide-processing enzymes. Blood. 79:2349-2355.

24. Hosaka, M., N. Masami, K. Won-Sin, W. Toshio, H. Kiyotaka, I. Jo, M. Kazuo, and N. Kazuhisa. 1991. Arg-X-Lys/Arg-Arg Motif as a signal for precursor cleavage catalyzed by Furin within the constitutive secretory pathway. J. Biol. Chem. 266:12127-12103.

25. Yoshimasa, Y., J. I. Paul, J. Whittaker, and D. F. Steiner. 1990. Effects of amino acid replacements within the tetrabasic cleavage site on the processing of the human insulin receptor precursor expressed in Chinese hamster ovary cells. J. Biol. Chem. 265:17230-17237.

26. Kawaoka, Y., and R. G. Webster. 1988. Sequence requirements for cleavage activation of influenza virus hemagglutinin expressed in mammalian cells. Proc. Natl. Acad. Sci. USA. 85:324-328.

27. Webster, R. G., and R. Rott. 1987. Influenza virus pathogenicity: the pivotal role of hemagglutinin. Cell. 50:665-666.

28. Walker, J. A., and Y. Kawaoka. 1993. Importance of conserved amino acids at the cleavage site of the haemagglutinin of a virulent avian influenza $A$ virus. J. Gen. Virol. 74:311-314.

29. Zhang, H., S. D. Apfelroth, W. Hu, E. C. Davis, C. Sangineti, J. Bonadio, R. P. Mecham, and F. Ramirez. 1994. Structure and expression of fibrillin-2, a novel microfibrillar component preferentially located in elastic matrices. J. Cell Biol. 124:855-863.

30. Barr, P. J. 1991. Mammalian subtilisins: the long-sought dibasic processing endoproteases. Cell. 66:1-3.

31. Stieneke-Grober, A., M. Vey, H. Angliker, E. Shaw, G. Thomas, C. Roberts, H. Klenk, and W. Garten. 1992. Influenza virus hemagglutinin with multibasic cleavage site is activated by furin, a subtilisin-like endoprotease. $E M B O$ (Eur. Mol. Biol. Organ.) J. 11:2407-2414.

32. Molloy, S. S., L. Thomas, J. K. VanSlyke, P. E. Stenberg, and G. Thomas. 1994. Intracellular trafficking and activation of the furin proprotein convertase: localization to the TGN and recycling from the cell surface. EMBO (Eur. Mol. Biol. Organ.) J. 13:18-33.

33. Klimpel, K. R., S. S. Molloy, G. Thomas, and S. H. Leppla. 1994. Anthrax toxin protective antigen is activated by a cell surface protease with the sequence specificity and catalytic properties of furin. Proc. Natl. Acad. Sci. USA. 89:1027710281 .

34. Herskowitz, I. 1987. Functional inactivation of genes by dominant negative mutations. Nature (Lond.). 329:219-222.

35. Byers, P. H. 1990. Brittle bones - fragile molecules: disorders of collagen gene structure and expression. Trends Genet. 8:293-300. 